\title{
On the structure of Stone lattices
}

\author{
P. D. Finch
}

C.C. Chen and G. Grätzer have shown that a Stone lattice is determined by a triple $(C, D, \phi)$ where $C$ is a boolean algebra, $D$ is a distributive lattice with 1 and $\phi$ is an e-homomorphism from $C$ into $D(D)$, the lattice of dual ideals of $D$.

It is shown here that any Stone lattice is, up to an isomorphism, a subdirect product of its centre $C(L)$ and a special Stone lattice $M(L)$. Special Stone lattices are characterised, in the terminology of the Chen-Grätzer triple, by the fact that the $e$-homomorphism $\phi$ is one to one.

In this paper we characterise a special Stone lattice $L$ as a triple $\left(H, C, D_{0}\right)$ where $H$ is a distributive lattice with 0 and $I, C$ is a boolean e-subalgebra of the centre of $H$ and $D_{0}$ is a sublattice of $H$ with $O$ such that

$$
d \in D_{0} \& c \in C \Rightarrow d \wedge c \in D_{0}
$$

and which separates the elements of $C$ in the sense that for any $c_{1} \neq c_{2}$ in $c$ there is a $d$ in $D_{0}$ with $d \leq c_{1}$ but $d \neq c_{2}$. It then turns out that $C$ is $C(L)$ and $D_{0}$ is the dual of $D(L)$.

\section{Introduction}

We adopt, without further explanation, the notation and terminology of Chen and Grätzer. The motivation for the results given below comes from Received 2 March 1970. 
PROPOSITION 1. Let $M$ be a stone lattice and let $\theta: B \rightarrow C(M)$ be an e-homomorphism from the boolean algebra $B$ onto the centre of $M$ which preserves complementation. Let $L$ be the set of ordered pairs $\langle x, b\rangle$, with $x$ in $M, b$ in $B$ and $b \theta=x^{* *}$. Then $L$ is $a$ subdirect product of $M$ and $B$, it is a Stone lattice, its centre $C(L)$ is isomorphic to $B$ and $D(L)=D(M) \times\{1\}$.

Proof. It is easily verified that

$$
\begin{aligned}
b_{i} \theta=x_{2}^{*}, i=1,2 \Rightarrow & \left(b_{1} \wedge b_{2}\right) \theta=\left(x_{1} \wedge x_{2}\right)^{* *} \& \\
\left(b_{1} \vee b_{2}\right) \theta= & \left(x_{1} \vee x_{2}\right) * * .
\end{aligned}
$$

It follows that $L$ is a sublattice of $M \times B$ and, since $\theta$ is onto $C(M), L$ is a subdirect product of $M$ and $B . L$ is distributive, it has a least element $\langle 0,0\rangle$ and a greatest element $\langle 1,1\rangle$. Since $\theta$ preserves complementation

$$
\langle x, b\rangle \in L \Rightarrow\left\langle x^{*}, b^{\prime}\right\rangle \in L
$$

where the prime denotes complementation in $B$. Since

$$
\langle x, b\rangle \wedge\langle y, c\rangle=\langle 0,0\rangle \Rightarrow\langle y, c\rangle \leq\left\langle x^{*}, b^{\prime}\right\rangle,
$$

$L$ is pseudocomplemented by

$$
\langle x, b\rangle^{*}=\left\langle x^{*}, b^{\prime}\right\rangle .
$$

Since

$$
\langle x, b\rangle * \vee\langle x, b\rangle * *=\left\langle x^{*} \vee x^{* *}, b \vee b^{*}\right\rangle=\langle 1,1\rangle,
$$

$L$ is a Stone lattice. Clearly

$$
D(L)=\{\langle d, I\rangle: d \in D(M)\}=D(M) \times\{1\}
$$

and

$$
C(L)=\{\langle x, b\rangle: x \in C(M), b \in B, b \theta=x\} .
$$

The correspondence $\langle x, b\rangle \rightarrow b$ and $b \rightarrow(b \theta, b\rangle$ are mutually inverse isomorphisms between $C(L)$ and $B$.

This proposition suggests the possibility that any Stone lattice $L$ is representable, up to an isomorphism, as a subdirect product of another suitably chosen Stone lattice $M$ and a boolean algebra $B$ isomorphic to the centre of $L$, in the manner described in the proposition. In what follows we show that this is so and we demonstrate it in the following 
way. We introduce a *-congruence $\tau$ on the stone lattice $L$ (i.e. a lattice congruence which preserves pseudocomplements). We say that a Stone lattice is special when the *-congruence $\tau$ on it is the identity relation. $L / \tau$ is a special stone lattice and $\tau_{0}$, the restriction of $\tau$ to $C(L)$, is a congruence on $C(L)$ which preserves complements, further $C(L / \tau)=C(L) / \tau_{0}$. The mapping $C(L)+C(L / \tau)$ is an e-homomorphism which preserves complementation and $L$ is isomorphic to a subdirect product of $L / \tau$ and $C(L)$ in the manner described in Proposition 1 with $L / \tau$ playing the role of $M$.

The *-congruence $\tau$ is defined explicitly by writing $x \tau y$ to mean (i) $\forall d \in D(L), d \geq x^{*} \Leftrightarrow d \geq y^{*}$, and

(ii) $x \vee x^{*}=y \vee y^{*}$.

Note that (ii) is always true when $x$ and $y$ are in $C(L)$.

Through the paper $L$ denotes a fixed but arbitrary Stone lattice, unless the contrary is explicitly stated.

\section{The congruence $\tau_{0}$ on $C(L)$}

For $a$ in $C(L)$ write

$$
G_{a}=\left\{d: d \in D(L), d \geq a^{*}\right\} .
$$

Then $a \tau_{0} b$ if and only if $G_{a}=G_{b}$. Then $\tau_{0}$ is an equivalence relation on $C(L)$, we write $a \tau_{0}$ for the $\tau_{0}$-equivalence class in $C(L)$ determined by $a, C(L) / \tau_{0}$ for the set of $\tau_{0}$-equivalence classes and $\tau_{0}^{4}$ for the natural mapping $C(L) \rightarrow C(L) / \tau_{0}$.

In fact $\tau_{0}$ is a congruence on $C(L)$ which preserves complements. That $\tau_{0}$ preserves join and meets follows from the fact that

$$
\begin{aligned}
G_{a \wedge b} & =G_{a} \wedge G_{b}, \\
G_{a \vee b} & =G_{a} \vee G_{b},
\end{aligned}
$$

where the lattice operations on the right are those of the lattice 
$D(D(L))$. From this it follows that $C(L) / \tau_{0}$ is a lattice with ordering

$$
a \tau_{0} \leq b \tau_{0} \text { if and only if } G_{a} \subseteq G_{b} \text {, }
$$

and lattice operations

$$
a \tau_{0} \wedge b \tau_{0}=(a \wedge b) \tau_{0}, a \tau_{0} \vee b \tau_{0}=(a \vee b) \tau_{0} .
$$

Note that

$$
0 \tau_{0}=\left\{a: a \in C(L), G_{a}=\{1\}\right\}
$$

is the least element of $C(L) / \tau_{0}$ and

$$
1 \tau_{0}=\left\{a: a \in C(L), G_{a}=D(L)\right\}
$$

is its greatest element.

Next we observe that $G_{a}$ is in the centre of $D(D(L))$, its complement being $G_{a^{*}}$, so that

$$
G_{a} \wedge G_{b}=G_{0} \Leftrightarrow G_{b} \subseteq G_{a^{*}}
$$

Thus

$$
a \tau_{0} \wedge b \tau_{0}=0 \tau_{0} \Leftrightarrow b \tau_{0} \leq a^{*} \tau_{0} \Leftrightarrow a \tau_{0} \leq b^{*} \tau_{0} .
$$

In particular

$$
a \tau_{0}=b \tau_{0} \Rightarrow b * \tau_{0} \leq a^{*} \tau_{0} \leq b^{*} \tau_{0}
$$

so that

$$
a \tau_{0} b \Longleftrightarrow a^{*} \tau_{0} b^{*}
$$

Thus one can define $\left(a \tau_{0}\right)^{*}=a^{*} \tau_{0}$. One verifies that $C(L) / \tau_{0}$ is a boolean algebra with complementation $a \tau_{0} \rightarrow\left(a \tau_{0}\right) *$.

We note that the congruence $\tau_{0}$ is just that associated with the structure map $\phi^{L}$ of Chen and Grätzer; this is an e-homomorphism from $C(L)$ into $D(D(L))$. We summarize the results above in

LEMMA 2.1. The map $\tau_{0}^{h}: C(L) \rightarrow C(L) / \tau_{0}$ is an e-homomorphism from 
the boolean algebra $C(L)$ onto the boolean algebra $C(L) / \tau_{0}$ which preserves complementation.

We conclude this section with

LEMMA 2.2. If $a \tau_{0} b$ then $d \vee a=d \vee b$ for every $d$ in $D(L)$.

Proof. $d \vee a$ is in $G_{a^{*}}=G_{b^{*}}$. Thus $d \vee a \geq d \vee b$, by symmetry $d \vee b \geq d \vee a$.

\section{3. $\tau$ is a *-congruence on $L$}

We start by providing some background motivation for the introduction of the *-congruence $\tau$. For $a$ in $C(L)$ let

$$
F_{a}=\left\{x: x \in L, x^{* *}=a\right\} \text {, }
$$

then as noted by Chen and Grä†zer, the correspondences

$$
x \rightarrow x \vee a^{*} \text { and } d \rightarrow d \wedge a
$$

are mutually inverse isomorphisms between $F_{a}$ and $G_{a}$. It follows that

LEMMA 3.1. If $a \tau_{0} b$ then the correspondences

$$
x \rightarrow\left(x \vee a^{*}\right) \wedge b \text { and } y \rightarrow\left(y \vee b^{*}\right) \wedge a
$$

are mutually inverse isomorphisms between the lattices $F_{a}$ and $F_{b}$.

Elements of $F_{a}$ and $F_{b}\left(a \tau_{0} b\right)$ which correspond under these isomorphisms will be said (provisionally) to be $\tau_{0}$-similar; more explicitly, elements $x, y$ in $L$ are $\tau_{0}$-similar when

(i) $x^{* *} \tau{ }_{0} y^{* *}$;

(ii) $\left(x \vee x^{*}\right) \wedge y^{* *}=y$ and $\left(y \vee y^{*}\right) \wedge x^{* *}=x$.

Then we have

LEMMA 3.2. Elements $x, y$ in $L$ are $\tau_{0}$-simizar if and only if they are $\tau$-equivalent.

Proof. T-equivalence clearly implies $\tau_{0}$-similarity. To prove the 
converse note that, since $y \vee y^{*}$ is dense, $x^{* *} \tau_{0} y^{* *}$ implies that $y \vee y^{*} \geq x^{*}$. From (ii) above we obtain

$$
x \vee x^{*}=\left(y \vee y^{*} \vee x^{*}\right) \wedge\left(x^{*} \vee x^{*}\right)=y \vee y^{*} \text {. }
$$

We mention the obvious.

\section{COROLLARY.}

1. $a, b \in C(L) \& a \tau_{0} b \Rightarrow a \tau b$

2. $a \in C(L), x \in L \& a \tau x \Rightarrow x \in C(L) \& a \tau_{0} x$;

3. $0 \tau=0 \tau_{0}, 1 \tau=1 \tau_{0}$.

We proceed now to show that $\tau$ is a *-congruence on $L$. That $\tau$ preserves meets and pseudocomplements is easy to prove and we dispose of it in

LEMMA 3.3 .

1. If $x \tau y$ then $x^{*} \tau y^{*}$;

2. if $x_{i} \tau y_{i}, i=1,2$ then $\left(x_{1} \wedge x_{2}\right) \tau\left(y_{1} \wedge y_{2}\right)$.

Proof. Firstly

$$
x \tau y=x^{* *} \tau_{0} y^{* *} \Rightarrow x^{*} \tau_{0} y^{*} \Rightarrow x^{*} \tau^{*} .
$$

Secondly, when $x_{i} \tau y_{i}$,

$$
\left(x_{1} \wedge x_{2}\right) * *=\left(x_{1}^{* *} \wedge x_{2}^{* *}\right)\left(y_{1}^{* *} \wedge y_{2}^{* *}\right)=\left(y_{1} \wedge y_{2}\right) * *
$$

and, using Lemma 2.2 at the third line below,

$$
\begin{aligned}
\left(x_{1} \wedge x_{2}\right) \vee\left(x_{1} \wedge x_{2}\right)^{*} & =\left\{\left(x_{1} \vee x_{1}^{*}\right) \wedge\left(x_{2} \vee x_{2}^{*}\right)\right\} \vee\left(x_{1} \wedge x_{2}\right)^{*} \\
& =\left\{\left(y_{1} \vee y_{1}^{*}\right) \wedge\left(y_{2} \vee y_{2}^{*}\right)\right\} \vee\left(x_{1} \wedge x_{2}\right)^{*} \\
& =\left\{\left(y_{1} \vee y_{1}^{*}\right) \wedge\left(y_{2} \vee y_{2}^{*}\right)\right\} \vee\left(y_{1} \wedge y_{2}\right)^{*} \\
& =\left(y_{1} \wedge y_{2}\right) \vee\left(y_{1} \wedge y_{2}\right)^{*} .
\end{aligned}
$$

This proves the lemna.

To show that $\tau$ preserves lattice joins is a little more difficult; we do so through a number of contributory results.

LEMMA 3.4. If $x$. $y$ and $d$ is in $D(L)$ then 


$$
d \vee x=d \vee y \text {. }
$$

Proof. By Lemma 2.2

$$
\begin{aligned}
x^{* *} \tau_{0} y^{* *} & \Rightarrow d \vee x^{* *}=d \vee y^{* *}, \\
x \tau y & \Rightarrow d \vee x \vee x^{*}=d \vee y \vee y^{*} .
\end{aligned}
$$

Then when $x \tau y$ one has

$$
\left(d \vee x^{* *}\right) \wedge\left(d \vee x \vee x^{*}\right)=\left(d \vee y^{* *}\right) \wedge\left(d \vee y \vee y^{*}\right)
$$

that is,

$$
d \vee x=d \vee y
$$

This is the desired result.

LEMMA 3.5. Write $d_{1}=x_{1} \vee x_{1}^{*}, d_{2}=x_{2} \vee x_{2}^{*}$; then

$$
x_{1} \vee x_{2} \vee\left(x_{1} \vee x_{2}\right)^{*}=\left(d_{1} \wedge d_{2}\right) \vee\left[\left(d_{1} \vee d_{2}\right) \wedge x_{1}^{* *} \wedge x_{2}^{*}\right] .
$$

Proof. Routine computation of the right-hand side.

We are now able to establish

LEMMA 3.6. If $x_{i} \tau y_{i}, i=1,2$; then $\left(x_{1} \vee x_{2}\right) \tau\left(y_{1} \vee y_{2}\right)$.

Proof. Clearly $\left(x_{1} \vee x_{2}\right)^{* *} \tau_{0}\left(y_{1} \vee y_{2}\right)^{* *}$. It is then only necessary to show that

$$
x_{1} \vee x_{2} \vee\left(x_{1} \vee x_{2}\right)^{*}=y_{1} \vee y_{2} \vee\left(y_{1} \vee y_{2}\right)^{*} .
$$

But this equation follows easily from Lemmas 3.4 and 3.5 since $\left(x_{1}^{* *} \wedge x_{2}^{* *}\right) \tau\left(y_{1}^{* *} \wedge y_{2}^{* *}\right)$ and $\tau$ preserves meets, so that

$$
\left[\left(d_{1} \vee d_{2}\right) \wedge x_{1}^{* *} \wedge x_{2}^{* *}\right] \tau\left[\left(d_{1} \vee d_{2}\right) \wedge y_{1}^{* *} \wedge y_{2}^{* *}\right] .
$$

Finally $d_{i}=x_{i} \vee x_{i}^{*}=y_{i} \vee y_{i}^{*}$ and so we obtain the desired result.

Collecting the above results we have

THEOREM 1. $L / \tau$ is a special Stone Zattice with pseudocomplementation $(x \tau)^{*}=x^{*} \tau, C(L / \tau)=C(L) / \tau_{0}$ and $D(L / \tau)$ is isomorphic to $D(L)$. Further $\tau_{0}: C(L) \rightarrow C(L / \tau)$ is an e-homomorphism of $C(L)$ onto $C(L / \tau)$ which preserves complementation.

That $L / \tau$ is a special Stone lattice is easily verified and the 
only part of the theorem which calls for proof is the statement about $D(L / \tau)$; to establish its isomorphism with $D(L)$ we need

\section{LEIMA 3.7 .}

(i) if $d_{1}, d_{2}$ are in $D(L)$ and $d_{1} \tau d_{2}$ then $d_{1}=d_{2}$,

(ii) if $d$ is in $D(L)$ then $d \tau$ is in $D(L / \tau)$,

(iii) if $x \tau$ is in $D(L / \tau)$ then there is exactly one $d$ in $D(L)$ with $x \tau=d \tau$.

Proof. If $d_{1}, d_{2}$ are in $D(L)$ and $d_{1} \tau d_{2}$ then

$$
d_{1}=d_{1} \vee a_{1}^{*}=d_{2} \vee a_{2}^{*}=d_{2} \text {. }
$$

If $d$ is in $D(L)$ then

$$
(d \tau) *=d^{*} \tau=0 \tau,
$$

so $d \tau$ is in $D(L / \tau)$.

Finally if $x \tau$ is in $D(L / \tau)$ then $(x \tau) *=0 \tau$ so

$$
\left(x \vee x^{*}\right) \tau=x \tau \vee x^{*} \tau=x \tau
$$

and $x \vee x^{*}$ is dense.

It follows from Lemma 3.7 that

$$
D(L / \tau)=\{d \tau: d \in D(L)\}
$$

and the isomorphism between $D(L / \tau)$ and $D(L)$ is an obvious consequence.

\section{4. $L$ is a subdirect product of $L / \tau$ and $C(L)$}

We prove

THEOREM 2. Let $L$ be a Stone lattice. Then $L$ is *-isomorphic with the subdirect product of $L / \tau$ and $C(L)$ consisting of alz ordered pairs $\langle t, a\rangle$ with $t$ in $L / \tau$, and $a$ in $C(L)$ and $a \tau_{0}=. t^{* *}$.

Proof. The theorem is proved by a routine verification that $\xi, \eta$ defined by

$$
\begin{gathered}
x \xi=\left\langle x \tau, x^{* *}\right\rangle, \quad x \in L, \\
\langle t, a\rangle \eta^{*}=t \wedge a, \quad a \tau_{0}^{\natural}=t^{* *},
\end{gathered}
$$


are mutually inverse *-isomorphisms between the two Stone lattices in question.

Thus any Stone lattice $L$ determines a triple $(C(L), M(L), \theta)$ where $M(L)=L / \tau$ is a special Stone lattice and $\theta$ is an e-homomorphism form $C(L)$ onto $C(M(L))$ which preserves complementation. Conversely, if $(B, M, \theta)$ is a triple where

(i) $B$ is a boolean algebra,

(ii) $M$ is a special Stone lattice,

(iii) $\theta$ is an e-homomorphism form $B$ onto $C(M)$ which preserves complementation,

then the Stone lattice $L$ constructed in Proposition $I$ is such that $B=C(L)$ and $M \simeq L / \tau$. This is the content of

PROPOSITION 2. If $M$ in Proposition 1 is a special Stone Zattice then $L / \tau$ is *-isomorphic to $M$.

Proof. Returning to the notation of Proposition 1 one finds that

$$
G_{\left\langle x^{* *}, b\right\rangle}=G_{x * *} X\{1\}
$$

and it follows that, if $M$ is a special stone lattice,

$$
\left\langle x^{* *}, b\right\rangle \tau_{0}\left\langle y^{* *}, c\right\rangle \Leftrightarrow x^{* *}=y^{* *} \text {. }
$$

Thus

$$
\begin{aligned}
\langle x, b\rangle \tau(y, c\rangle & \Rightarrow x^{* *}=y^{* *} \& x \vee x^{*}=y \vee y^{*} \\
& \Rightarrow x=\left(x \vee x^{*}\right) \wedge x^{* *}=\left(y \vee y^{*}\right) \wedge y^{* *}=y .
\end{aligned}
$$

Hence, for $\langle x, b\rangle$ in $L$, one has

$$
\langle x, b\rangle \tau=\{\langle x, c\rangle: c \theta=b \theta\} \text {. }
$$

It is now routine to verify that the correspondence $x \rightarrow\langle x, b\rangle \tau$ is a *-isomorphism between $M$ and $L / \tau$.

The preceeding results reduce the study of arbitrary Stone lattices to that of special stone lattices. As noted in the summary, special stone lattices are characterised by the fact that the structure map in their Chen-Grätzer triple is one to one. It follows at once that a special Stone lattice $M$ is characterised by the distributive lattice $D(M)$ and 
a boolean e-subalgebra of the centre of $D(D(M))$, namely the image of the lattice $C(M)$ under the structure map $\phi$. However, rather than pursue a direct analogy with the approach of Chen and Grätzer we embark on a slightly more oblique one in the next section. This second approach is based on the observation that the sublattice of $D(D(M))$ consisting of the principal dual ideals of $D(M)$ is isomorphic with the dual of $D$. We can then characterise a special stone lattice through the distributive lattice $D(D(M))$, a boolean e-subalgebra of its centre and $P(D(M))$, the sublattice of principal dual ideals of $D(M)$.

\section{The construction of Stone lattices}

We prove the following

THEOREM 3. Let $B$ be a distributive lattice with 0 and 1 . Let $C$ be a boolean e-subalgebra of $H$ and let $D_{O}$ be a sublatice of $H$ which contains 0 and which has the property that

$$
d \in D_{0} \& c \in C \Rightarrow d \wedge c \in D_{0} \text {. }
$$

Let $L$ be the set of ordered pairs $\langle d, c\rangle$ with $d$ in $D_{0}, c$ in $c$ and $d \leq c$. Order $L$ by the prescription $\left(d_{1}, c_{1}\right) \leq\left\langle d_{2}, c_{2}\right\rangle$ if and only if $c_{1} \leq c_{2}$ and $d_{2} \wedge c_{1} \leq d_{1}$.

Then $(L, \leq)$ is a lattice which is a Stone lattice pseudocomplemented by

$$
\langle d, c\rangle *=\left\langle 0, c^{\prime}\right\rangle,
$$

the prime denoting complementation in $C$. Its centre is isomorphic to $C$ and its lattice of dense elements is isomorphic to the dual of $D_{0}$. Moreover $(L, \leq, *)$ is a special Stone lattice if and only if $D_{0}$ separates the elements of $c$, in the sense that for any $c_{1} \neq c_{2}$ in $C$ there is $d$ in $D_{0}$ with $d \leq c_{1}$ but $d \neq c_{2}$.

Proof. S is clearly reflexive and antisymetric on $L$. To establish transitivity we note 


$$
\begin{array}{r}
\left\langle d_{1}, c_{1}\right\rangle \leq\left\langle d_{2}, c_{2}\right\rangle \leq\left\langle d_{3}, c_{3}\right\rangle \Rightarrow c_{1} \leq c_{2} \leq c_{3} \& c_{1} \wedge d_{2} \leq d_{1} \& \\
\quad c_{2} \wedge d_{3} \leq d_{2} \\
\quad c_{1} \leq d_{3} \& c_{1} \wedge d_{3}=c_{1} \wedge c_{2} \wedge d_{3} \leq c_{1} \wedge d_{2} \leq d_{1} .
\end{array}
$$

To establish the lattice property we show that

(1) $\left\langle d_{1}, c_{1}\right\rangle \wedge\left\langle d_{2}, c_{2}\right\rangle=\left\langle\left(d_{1} \wedge c_{2}\right) \vee\left(d_{2} \wedge c_{1}\right), c_{1} \wedge c_{2}\right\rangle$,

(2) $\left(d_{1}, c_{1}\right\rangle \vee\left\langle d_{2}, c_{2}\right\rangle=\left\langle\left(d_{1} \wedge c_{2}^{\prime}\right) \vee\left(d_{2} \wedge c_{1}^{\prime}\right) \vee\left(d_{1} \wedge d_{2}\right), c_{1} \vee c_{2}\right)$.

To prove ( 1 ), one verifies firstly that its right-hand side is in $L$ and is a common lower bound to $\left\langle d_{1}, c_{1}\right\rangle,\left\langle d_{2}, c_{2}\right\rangle$; if $\left\langle d_{3}, c_{3}\right\rangle$ in $L$ is any other lower bound one has $d_{3} \leq c_{3} \leq c_{1} \wedge c_{2}$ and $c_{3} \wedge\left(d_{1} \vee d_{2}\right) \leq d_{3}$. Then

$$
c_{3} \leq\left\{\left(d_{1} \vee d_{2}\right) \wedge c_{1} \wedge c_{2}\right\} \leq d_{3}
$$

so

$$
\left\langle\bar{d}_{3}, c_{3}\right\rangle \leq\left\langle\left(d_{1} \vee d_{2}\right) \wedge c_{1} \wedge c_{2}, c_{1} \wedge c_{2}\right\rangle .
$$

Since

$$
\left(d_{1} \vee d_{2}\right) \wedge c_{1} \wedge c_{2}=\left(d_{1} \wedge c_{2}\right) \vee\left(d_{2} \wedge c_{1}\right),
$$

we obtain the desired result.

To establish (2) one verifies firstly that its right-hand side is in $L$ and is a common upper bound to $\left\langle d_{1}, c_{1}\right\rangle,\left\langle d_{2}, c_{2}\right\rangle$. On the other hand if $\left\langle d_{3}, c_{3}\right\rangle$ in $L$ is any other upper bound then $d_{3} \vee c_{1} \vee c_{2} \leq c_{3}$ and $a_{3} \wedge c_{i} \leq d_{i}, i=1,2$. Whence

$$
d_{3} \leq\left(d_{1} \vee c_{1}^{\prime}\right) \wedge\left(d_{2} \vee c_{2}^{\prime}\right) \text {. }
$$

From

$$
\begin{aligned}
\left(d_{1} \vee c_{1}^{\prime}\right) \wedge\left(d_{2} \vee c_{2}^{\prime}\right) \wedge\left(c_{1} \vee c_{2}\right), c_{1} \vee c_{2} & \leq\left\langle d_{3} \wedge\left(c_{1} \vee c_{2}\right), c_{1} \vee c_{2}\right\rangle \\
& \leq\left\langle d_{3}, c_{3}\right\rangle
\end{aligned}
$$

and

$$
\left(d_{1} \vee c_{1}^{\prime}\right) \wedge\left(d_{2} \vee c_{2}^{\prime}\right) \wedge\left(c_{1} \vee c_{2}\right)=\left(d_{1} \wedge c_{2}^{\prime}\right) \vee\left(d_{2} \wedge c_{1}^{\prime}\right) \vee\left(d_{1} \wedge d_{2}\right)
$$

we obtain the desired result.

It is routine, though tedious, to verify that $L$ is distributive. Straight-forward calculations establish that, $\langle 0,0\rangle$ is the least element in $L,\langle 0,1\rangle$ is the greatest element, 


$$
\left\langle d, d^{*}=\left\langle 0, c^{\prime}\right\rangle,\right.
$$

and that $L$ is a Stone lattice and finally that

$$
\begin{aligned}
& C(L)=\{\langle 0, c\rangle: c \in C\} \\
& D(L)=\left\{\langle d, I\rangle: d \in D_{0}\right\} .
\end{aligned}
$$

To establish the conditions under which $L$ is a special Stone lattice observe that

$$
\langle d, 1\rangle \geq\left\langle 0, c^{\prime}\right\rangle \Leftrightarrow d \wedge c^{\prime}=0 .
$$

Since $d \wedge c$ and $d \wedge c^{\prime}$ belong to $D_{0}$

$$
d=(d \wedge c) \vee\left(d \wedge c^{\prime}\right)
$$

and we deduce that

$$
\left\langle 0, c_{1}\right\rangle \tau_{0}\left\langle 0, c_{2}\right\rangle \Leftrightarrow " \forall d \in D_{0}, d \leq c_{1} \Leftrightarrow d \leq c_{2} " .
$$

But if $L$ is a special Stone lattice $\tau_{0}$ is the identity relation on $C(L)$ and we have the property stated in the theorem. Conversely if that property holds then $\tau_{0}$ is the identity relation on $C(L)$. If then $\left\langle d_{1}, c_{1}\right\rangle \tau\left\langle d_{2}, c_{2}\right\rangle$ we must have $c_{1}=c_{2}=c$, say, and

$$
\left\langle d_{1}, 1\right\rangle=\left\langle d_{1}, c\right\rangle \vee\left\langle d_{1}, c\right\rangle^{*}=\left\langle d_{2}, c\right\rangle \vee\left\langle d_{2}, c\right\rangle^{*}=\left\langle d_{2}, 1\right\rangle
$$

and so $\tau$ is the identity relation on $L$, that is $L$ is a special Stone lattice.

We show now that any special Stone lattice can be constructed in the way described in Theorem 3 .

THEOREM 4. Let $L^{\prime}$ be a special Stone lattice. Take $H=D\left(D\left(L^{\prime}\right)\right) D_{0}=P\left(D\left(L^{\prime}\right)\right)$, the set of principal dual ideals of $D\left(L^{\prime}\right)$, and let $C$ be a boolean e-subalgebra of $C\left(D\left(D\left(L^{\prime}\right)\right)\right)$. Then $L^{\prime}$ and $L$ are isomorphic as Stone lattices.

Proof. We firstly verify that $L$ is a special Stone lattice. This follows from the fact that the intersection of any principal dual ideal of a distributive lattice with an arbitrary dual ideal in the centre of the lattice of its dual ideals is again a principal dual ideal. Thus 


$$
d \in P\left(D\left(L^{\prime}\right)\right) \& c \in C\left[D\left(D\left(L^{\prime}\right)\right)\right] \Rightarrow d \wedge c \in P\left(D\left(L^{\prime}\right)\right)
$$

That $D_{0}$ separates $C$ is obvious since $L^{\prime}$ is special.

The theorem is proved by verifying that the correspondence

$$
x \rightarrow\left(\left[x \vee x^{*}\right), G_{x^{* *}}\right)
$$

from $L^{\prime}$ onto $L$ is the desired isomorphism.

\section{Reference}

[1] C.C. Chen and G. Grätzer, "Stone lattices. I: Construction theorems", Canad. J. Math. 21 (1969), 884-894.

Monash University,

clayton, victoria. 